\title{
Availability Analyses of Virtual Satellite Network Attachment Point Constructed by Satellite-fixed Footprints Technique
}

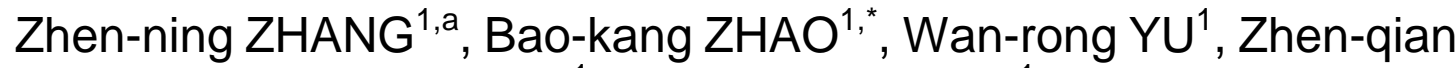 \\ FENG $^{1}$ and Chun-qing $\mathrm{WU}^{1}$ \\ ${ }^{1}$ College of Computer, National University of Defense Technology, China \\ azhangzhenning12@nudt.edu.cn \\ ${ }^{*}$ Corresponding author
}

Keywords: Satellite networks, Virtual attachment point, Satellite-fixed footprints.

\begin{abstract}
Virtual satellite network attachment point approach is explored to hide the mobility of satellites from the mobile endpoints in the highly dynamic NGEO satellite network. In this work, a mathematical model for the satellite-fixed footprint technique and a new evaluation criterion are introduced to analyze the availability of a virtual attachment point. Analyzing the quantitative evaluation results, we conclude with the benefits and some drawbacks of constructing a satellite-fixed footprint based virtual attachment point.
\end{abstract}

\section{Introduction}

Connecting the remaining two-thirds of the humankind that currently does not have access to the Internet in a cost effective manner, satellite network is an attractive option to provide broadband integrated Internet services to globally scattered mobile users [1]. Non-geostationary (NGEO) systems consisting of Medium Earth Orbit (MEO) and Low Earth Orbit (LEO) satellites offer lower latency, lower free space loss, and better re-use of available ground-space communication frequencies, but these advantages come with a price: lower orbit satellites move with higher speeds relative to the earth's surface, resulting in high dynamic in the satellite network topology. This topological phenomenon constitutes a major challenge in providing efficient service for rapidly growing mobile user [2]. Therefore, enhancing mobility in a highly dynamic NGEO satellite network is widely explored in satellite networks research.

To address this issue, logically fixed virtual satellite network attachment points [3] are superimposed over the physical network in order to hide the mobility of satellites from the mobile endpoints. A fixed virtual attachment point regularly embodied by different satellites is considered as a logical network location (decided from its location in the network topology). Then the proposal enhances endpoint mobility by a separation of identity and logical network location through an identity-to-location resolution [4] along with taking advantage of the user's geographical location information. Building a virtual attachment point is a task involving a variety of technical challenges.

In this work, a mathematical model for the satellite-fixed footprint technique and a new evaluation criterion are introduced to analyze the availability of virtual attachment point. Comparing the quantitative evaluation results, we conclude with the benefits and drawbacks of constructing a satellite-fixed footprint based virtual attachment point.

The paper is organized as follows. In Section II we explain the satellite-fixed footprint based virtual attachment point scheme, and in Section III we describe the mathematical model for the satellite-fixed footprint technique and the new evaluation 
criterion. In Section IV we present availability evaluation of the satellite-fixed footprint based virtual attachment point and in Section V we conclude the paper.

\section{Satellite-Fixed Footprint Based Virtual Attachment Point}

In this paper, we discuss a NGEO satellite network which is poised to be an integral part of the future global communications infrastructure [1]. In the terrestrial mobile networks, only endpoints are subject to motion while network attachment points remain fix. But in NGEO satellite networks, both endpoints and satellites keep on moving. Therefore, a main issue is that relative movement between a mobile endpoint and a satellite makes the access device's network address corresponding to the current point of attachment of the satellite network object becoming elusive.

A proposal [3] that helps achieve the goal of enhanced mobility is a logical separation of the movement of satellites and the movement of endpoints, which is achieved by introducing the concept of virtual attachment points. A fixed virtual attachment point regularly embodied by different satellites is considered as a logical network location (decided from its location in the network topology). Then the proposal enhances endpoint mobility by a separation of identity and logical network location through an identity-to-location resolution.

Exploiting geographical location information provided by the GPS and continually tracking the mobility of endpoints, the global location resolution service dynamically binds endpoints to its virtual attachment point (encoded geographical coordinates or a specific service scope number according to different location management strategies). The main task of constructing a virtual attachment point is building a certain association between a virtual attachment point and the satellites embodying it.

Satellite-fixed footprint technique [5] is generally appropriate for satellite with nadir pointing antenna systems. As the satellite moves along the orbit, its footprint sweeps across the surface with a constant velocity. Considering the periodic and predictable movement of satellite and the definite boundary of satellite coverage, a binding between a virtual attachment point and the satellites is simply determined. However, we still need to evaluate the availability of satellite-fixed footprint based scheme.

\section{Availability Analyses of Satellite-fixed Footprints Based Scheme}

A single satellite may not be sufficient to serve all endpoints connected to the virtual attachment point due to high service demand of mobile data traffic or due to shadowing by terrain and buildings. Availability can be increased by binding a virtual attachment point with multiple satellites [6].

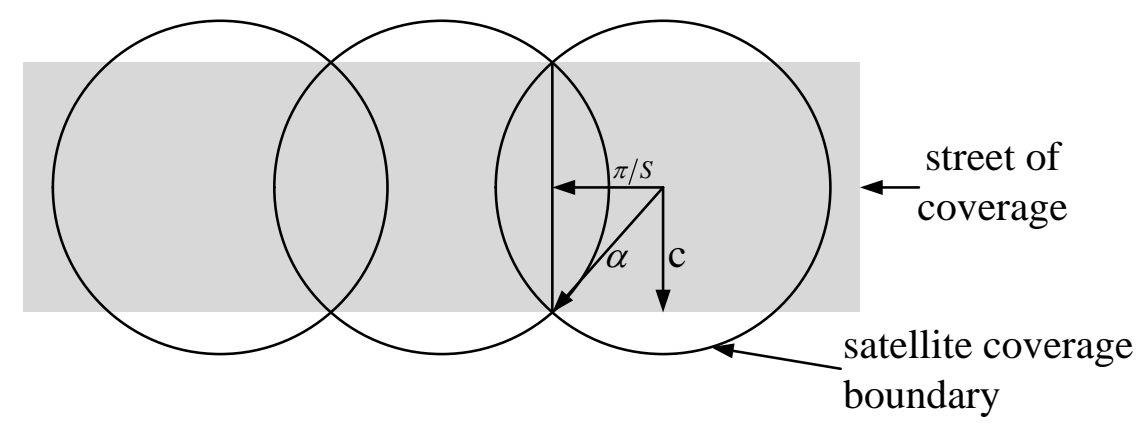

Figure 1. Illustration of street of coverage 
Therefore, in our evaluation, the availability of a virtual attachment point is simply defined as average number of satellites per footprint area, which is equal to, in numeral, the average number of satellites per unit angular length.

In order to provide a formal description, we give set of definitions and presupposition. In our analysis of virtual attachment point availability aspect, we consider a polar satellite constellation with $P$ orbits and each of which has $S$ identical satellites. We also assume that inter-satellite angular distances are equal for the satellites in the same orbit. And then we give the definition namely street of satellite coverage [7]. For polar satellite constellation capable of providing world-wide coverage, each orbital plane includes enough satellites to provide a continuous street of coverage within a certain band. As shown in Fig. 1, the geocentric angular width of a street of coverage $c$ can be expressed by

$$
c=\arccos \left(\frac{\cos \alpha}{\cos (\pi / S)}\right) \text {, }
$$

where $\alpha$ is the semi-geocentric angle of each satellite coverage, and $\pi / S$ is the semi-geocentric angular length between satellites in the same orbit.

To simplify the analysis, we also assume that the geocentric angular distances are equal between different orbits. But because of the special structure, the distribution of satellites in a polar orbit satellite constellation is not homogeneous. The geocentric angular length between adjacent orbits has maximum value at the equator and decreases towards the poles. Therefore, when considering the target of seamless coverage in a global range, we consider only how to achieve continuous coverage of the equator, i.e.,

$$
P \times 2 c \geq \pi
$$

We consider effective footprint of a satellite as a one-dimensional circular segment whose angular length is equivalent to the geocentric angular width of the street of coverage c, i.e.,

$$
L_{f p}(l a t=0)=2 c=2 \arccos \left(\frac{\cos \alpha}{\cos (\pi / S)}\right) .
$$

And the number of footprint areas along the equator is calculated as

$$
N_{f p}(\text { lat }=0)=\frac{\pi}{\arccos (\cos \alpha / \cos (\pi / S))} \text {. }
$$

Then the average number of satellites per footprint area is the following:

$$
N_{s a t / f p}^{a v g}(l a t=0)=\frac{N_{s a t}}{N_{f p}(\text { lat }=0)}=\frac{2 P \times \arccos (\cos \alpha / \cos (\pi / S))}{\pi} .
$$

According to the geometry of for world-wide coverage by NGEO satellites using satellite-fixed footprint technique, for arbitrary latitude lat, we have

$$
L_{f p}(\text { lat })=\arcsin \left(\frac{\sin \left(L_{f p}(\text { lat }=0)\right)}{\cos (\text { lat })}\right)=\arcsin \left(\frac{\sin (2 \arccos (\cos \alpha / \cos (\pi / S)))}{\cos (l a t)}\right)
$$
and 


$$
N_{f p}(\text { lat })=\frac{2 \pi}{L_{f p}(\text { lat })}=\frac{2 \pi}{\arcsin (\sin (2 \arccos (\cos \alpha / \cos (\pi / S))) / \cos (\text { lat }))} .
$$

Then

$$
N_{\text {sat } / f p}^{\text {avg }}(\text { lat })=\frac{N_{\text {sat }}}{N_{f p}(\text { lat })}=\frac{P \times \arcsin (\sin (2 \arccos (\cos \alpha / \cos (\pi / S))) / \cos (\text { lat }))}{\pi} .
$$

\section{Availability Evaluation of Satellite-fixed Footprints Based Scheme}

For simplicity, we consider the scene of minimum coverage which means satellites along the equator just exactly cover the equator areas without overlapping. Therefore, we set $P \times 2 c=\pi$. Then we have

$$
L_{f p}(l a t=0)=2 c=\pi / P
$$

and

$$
N_{f p}(\text { lat }=0)=2 \pi / 2 c=2 P .
$$

And then

$$
N_{\text {sat } / f p}^{a v g}(l a t=0)=\frac{N_{s a t}}{N_{f p}(l a t=0)}=1
$$

For arbitrary latitude lat, we have

$$
L_{f p}(\text { lat })=\arcsin \left(\frac{\sin \left(L_{f p}(\text { lat }=0)\right)}{\cos (\text { lat })}\right)=\arcsin \left(\frac{\sin (\pi / P)}{\cos (\text { lat })}\right)
$$

and

$$
N_{f p}(\text { lat })=\frac{2 \pi}{L_{f p}(\text { lat })}=\frac{2 \pi}{\arcsin (\sin (\pi / P) / \cos (\text { lat }))} .
$$

Then

$$
N_{\text {sat } / f p}^{a v g}(\text { lat })=\frac{N_{s a t}}{N_{f p}(\text { lat })}=\frac{P \times \arcsin (\sin (\pi / P) / \cos (\text { lat }))}{\pi} .
$$

The average number of satellites per footprint area are illustrated in Fig. 2 for various lat values when we set the value of $\mathrm{P}$ to 4 and 12, corresponding to a MEO satellite system and a LEO satellite system, respectively. 


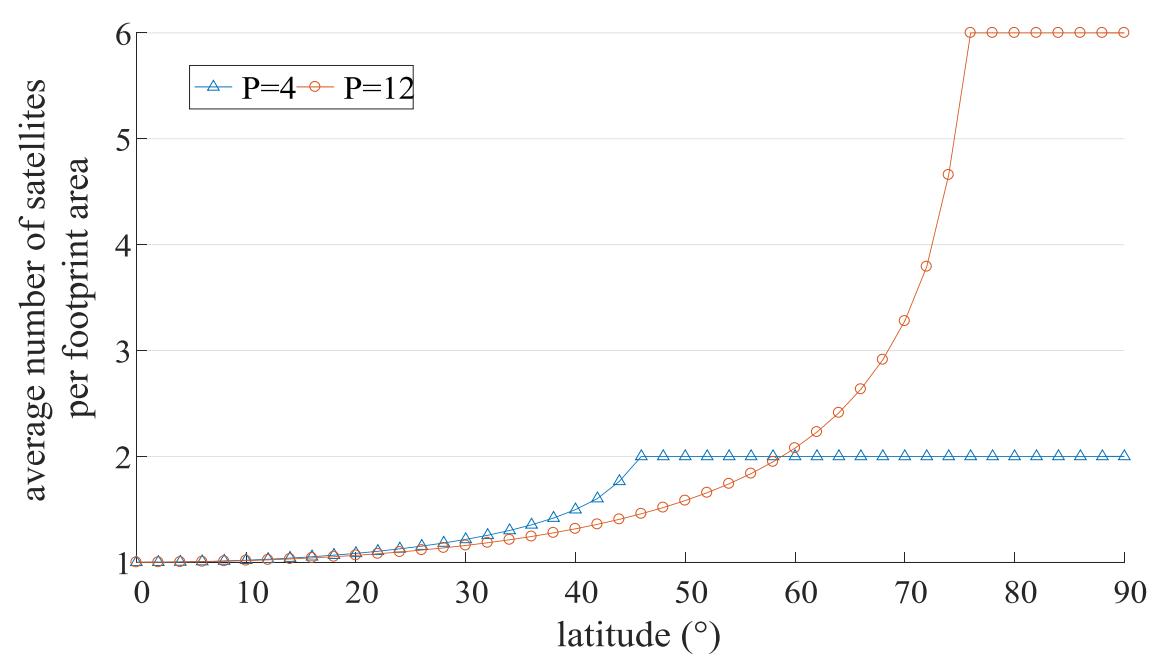

Figure 2. Average number of satellite per footprint area $(\mathrm{P}=4$ and $\mathrm{P}=12)$

As shown in Fig.2, the average number of satellites per footprint area, representing the availability of a virtual attachment point, are continuously increases with the latitude until they reach the maximum values. In the equatorial and low latitude regions, the simulated value is close without significant difference, while in mid-latitude the MEO satellite system with smaller number of satellites offers better availability than the LEO satellite system with more satellites, but in the polarization LEO satellite system performs better than MEO satellite system. Note that the total availability of the overall virtual attachment points constructed by larger scale LEO satellite system is more excellent than the MEO solution which demonstrates the theoretical analysis and mathematical derivation above.

Analyzing from the simulation and the test result, large scale LEO satellite system is more promising to be the basis for constructing the high available virtual attachment points. The simulation of the global distribution of availability also reflects the basic coverage nature of satellite-fixed footprint based polar orbit satellite system, but this availability distribution does not correspond to the worldwide population distribution and the satellite broadband market demand (specifically is latitudinal summed across all longitudes). Moreover, the continuous changing of availability along the latitude will inevitably bring barriers to guarantee QoS, and even leads to frequent handover between virtual attachment points and physical satellites. The optimization of these issues can be investigated in the following work utilizing the hybrid satellite system or earth-fixed satellite footprint technique.

\section{Conclusions}

As one of the most convenient and easy deployment of the technology, satellite-fixed footprint technique has long been one of the main stream satellite footprint scheme. The purpose of this paper is to present a mathematical description of the availability of virtual attachment points, for the sake of evaluating the satellite-fixed footprint based scheme. And the availability of a virtual attachment point is simply defined as average number of satellites per footprint area. By analyzing the model and simulation result, we conclude that the latitudinal distribution of availability, and some deficiencies, which come from the nature of satellite-fixed footprint technique as well as the polar orbit satellite constellation. 


\section{Acknowledgement}

This research was financially supported by the National Science Foundation of China No.61202488, No.61379147, No.61103182.

\section{References}

[1] F. Khan, Mobile Internet from the Heavens, Computer Science. (2015).

[2] Cisco, Visual networking index: global mobile data traffic forecast update, pages 2015-2020, White paper. (2016).

[3] Zhang, Z., Zhao, B., Feng, Z., Yu, W., and Wu, C., MSN: a mobility-enhanced satellite network architecture: poster, Proceedings of the 22nd Annual International Conference on Mobile Computing and Networking. (2016) 465-466.

[4] Sharma, A., Tie, X., Uppal, H., Venkataramani, A., Westbrook, D., \& Yadav, A., A global name service for a highly mobile internetwork, ACM SIGCOMM Computer Communication Review. (2014) 247-258.

[5] Korcak, O., Alagoz, F., Multi-state virtual network architecture for next generation satellite networks, IEEE GLOBECOM 2007-IEEE Global Telecommunications Conference. (2007) 5031-5036.

[6] Korçak, Ö., \& Alagöz, F., Virtual topology dynamics and handover mechanisms in Earth-fixed LEO satellite systems, Computer networks. (2009) 1497-1511.

[7] Chan, S., Samuels, A. T., Shah, N. B., Underwood, J. E., and De Weck, O. L., Optimization of Hybrid Satellite Constellations using Multiple Layers and Mixed Circular-Elliptical Orbits, 22nd AIAA International Communications Satellite Systems Conference and Exhibit. (2004) 9-12. 